\title{
Asymptotic Probability Bounds on the Peak Distribution of Complex Multicarrier Signals Without Gaussian Assumption*
}

\author{
Masoud Sharif and Babak Hassibi \\ Department of Electrical Engineering \\ California Institute of Technology \\ Pasadena, CA, 91125
}

\begin{abstract}
Multicarrier signals exhibit a large peak to mean envelope power ratio (PMEPR). In this paper, we derive lower and upper probability bounds for the PMEPR distribution when entries of the codeword, $C$, are chosen independently from a symmetric q-ary PSK or QAM constellation, $C \in \mathcal{Q}_{q}^{n}$, or $C$ is chosen from a complex $n$ dimensional sphere, $\Omega_{n}$ when the number of subcarriers, $n$, is large and without any Gaussian assumption on either the joint distribution or any sample of the multicarrier signal. Even though the worst case PMEPR is of the order of $n$, the main result is that the PMEPR of a random codeword $C$ chosen from $\mathcal{Q}_{q}^{n}$ or $\Omega_{n}$ is $\log n$ with probability one, asymptotically. We then obtain a Varsharmov-Gilbert (VG) style bound for the achievable rate and minimum Hamming distance of codes chosen from $\mathcal{Q}_{q}^{n}$, with PMEPR of less than $\log n$. It is proved that asymptotically, the $V G$ bound remains the same for codes chosen from $\mathcal{Q}_{q}^{n}$ with PMEPR of less than $\log n$.
\end{abstract}

\section{Introduction}

Multicarrier modulation has been proposed in different wireless and wireline applications such as wireless local area networks (WLAN) and digital subscriber line (DSL). Even though multicarrier modulation has a very good performance in a multipath fading environment, it suffers from high amplitude variation which is unfavorable from a practical point of view. Different schemes have been proposed to reduce the peak to mean envelope power ratio (PMEPR) such as coding methods, clipping, dummy carriers, and selective mapping (SLM) $[1,2,3,4,5,6]$.

Clearly, the worst case peak to mean envelope power

*This work was supported in part by the National Sience Foundation uder grant no. CCR.0133818, by the office of Naval Research under grant no. N00014-02-1-0578, and by Caltech's Lee Center for Advanced Networking ratio (PMEPR) of multicarrier signals is of the order of $n$ where $n$ is the number of subcarriers. On the other hand, the numerical evaluation of the distribution of PMEPR shows that encountering $n$ as the worst case is highly unlikely [7]. This in fact motivates the problem of finding the PMEPR distribution to quantify how severe is that. In [7], by assuming that the multicarrier signal is a Gaussian process, an expression for the probability distribution of PMEPR is derived. This is a very strong assumption which is mathematically not valid when the modulating information is chosen from QAM/PSK constellation or from a complex $n$ dimensional sphere. On the other hand, by only assuming that each sample of the multicarrier signal has a Gaussian distribution, an upper bound for the complementary cumulative distribution function of PMEPR has been derived in [8].

In this paper for large $n$, we derive upper and lower bounds for the PMEPR distribution when the modulating codewords are chosen from symmetric QAM/PSK constellations or from the $n$ dimensional complex sphere without any Gaussian assumption either on each sample or on the whole process. The bounds are a generalization of the result of Halasz for Littlewood trigonometric polynomials to any symmetric complex constellation and also to spherical codes for polynomials over the unit circle [9]. We then use these bounds to determine the achievable rate of codes with bounded PMEPR.

To summarize the results, we show that with probability one, any codeword either with entries chosen independently from a symmetric QAM/PSK constellation or chosen uniformly from a complex sphere has PMEPR of $\log n+O(\log \log n)$ for a large number of subcarriers. Based on this result, we determine the achievable rate of codes with bounded PMEPR. We prove that there exist codes with high rate and large minimum Hamming distance as long as the PMEPR is less than $\log n$ for sufficiently large $n$.

The rest of the paper is outlined as follows. Section 2 in- 
troduces the notation, multicarrier signals, and the PMEPR of a codeword. The lower and upper probability bounds for the PMEPR distribution are derived in Section 3. In Section 4 we discuss the consequences of the bounds and we obtain a Varsharmov-Gilbert type bound for the achievable rate of codes with bounded PMEPR and with given minimum Hamming distance. Finally, Section 5 concludes the paper with further results and open problems.

\section{Definitions}

The normalized complex envelope of a multicarrier signal with $n$ subcarriers may be written as

$$
s_{C}(\theta)=\sum_{i=1}^{n} c_{i} e^{j \theta i}, \quad 0 \leq \theta<2 \pi,
$$

where $C=\left(c_{1}, \ldots, c_{n}\right)$ is the complex modulating vector with entries from a given complex constellation $\mathcal{Q}$. The admissible modulating vector is called codeword and the ensemble of all possible codewords constitute the code $\mathcal{C}$. The peak to mean envelope power ratio (PMEPR) of each codeword $C$ is defined as,

$$
\operatorname{PMEPR}_{C}(C)=\max _{0 \leq \theta<2 \pi} \frac{\left|s_{C}(\theta)\right|^{2}}{E\left\{\|C\|^{2}\right\}} .
$$

Similarly, PMEPR $R_{C}$ is defined as the maximum of (2) over all codewords in $\mathcal{C}$. In this paper, we will consider two classes of codes, namely, complex symmetric $q$-ary codes in which each coordinate is chosen from a complex symmetric constellation including QAM and PSK with $q$ alphabets, $C \in \mathcal{Q}_{q}^{n}$, and spherical codes. By symmetry, we mean that the probability distribution function for the constellation is even, equivalently, each point $A$ has the same probability as $-A$ in the constellation.

On the other hand, codewords of a spherical code are points on a complex $n$ dimensional sphere defined as,

$$
\Omega_{n}=\left\{\left(c_{1}, \ldots, c_{n}\right) \in C^{n}: \sum_{i=1}^{n}\left|c_{i}\right|^{2}=n\right\}
$$

It is worth noting that $E\left\{\|C\|^{2}\right\}=n E_{a v}$ for random $q$-ary codes where $E_{a v}$ is the average energy of the constellation $\mathcal{Q}_{q}$. Also, for spherical codes chosen from $\Omega_{n}, E\left\{\|C\|^{2}\right\}=n$ since all the codewords have constant norm.

It is clear from the definition of PMEPR that if all the carriers add up coherently, PMEPR can be of the order of $n$. However numerical results suggest that this barely occurs. The main goal of this paper is to get a better insight to the distribution of PMEPR.

Throughout the paper we use the following notations: $\mathcal{C}$ and $C$ represent a code family and a codeword, $c_{i}$ denotes the $i$ 'th coordinate of the codeword $C$, and $\log n$ is the natural logarithm. We use $\alpha$ and $\beta$ as arbitrary constants and $f(n)=O(g(n))$ denotes that $\lim _{n \rightarrow \infty}\left|\frac{f(n)}{g(n)}\right| \leq|\alpha|$.

\section{Bounds for Cumulative Distribution Func- tion of PMEPR}

In this section, we consider codewords are either chosen from $\mathcal{Q}_{q}^{n}$ or from $\Omega_{n}$. It is a commonly assumed in the literature that when the $c_{i}$ 's are independently chosen, $s_{C}(\theta)$ can be approximated as a Gaussian process [7]. However, this is not mathematically rigorous for spherical codewords and codewords with entries from $\mathcal{Q}_{q}$. In other words, by assuming that the $c_{i}$ 's are independently and identically distributed, even though it is conceivable that any finite samples of $s_{C}(\theta)$ is jointly Gaussian for large values of $n$, this statement is not valid for $n$ samples of $s_{C}(\theta)$.

In this section without using any Gaussian assumption, we derive upper and lower bounds for PMEPR distribution for different schemes. Derivation of the bounds are the generalization of the result of Halasz, for the asymptotic distribution of the maximum of $\sum_{i=1}^{n}\left|a_{i} \cos (i \theta)\right|$ when $a_{i}$ 's are chosen from $\{+1,-1\}$ with equal probability, to polynomials over the unit circle with coefficient chosen from $\mathcal{Q}_{q}$ as well as spherical codes.

\subsection{Upper Bound}

In the following theorem, we find an upper bound for the probability of having a PMEPR slightly larger than $\log n$ and we show that asymptotically this probability goes to zero. Theorem 1 is for the symmetric QAM constellations and it can be generalized to symmetric PSK and spherical codes as well [10].

Theorem 1 (Upper Bound-QAM Case), Let $s_{C}(\theta)=$ $\sum_{i=1}^{n} c_{i} e^{j 2 \pi \theta i}$ where $c_{i}=a_{i}+j b_{i}$ and the $a_{i}$ 's and $b_{i}$ 's each have i.i.d and even distributions. Also, let $\mathcal{C}_{q}^{Q A M}$ be the ensemble of all the admissible codewords. Then, for large values of $n$, and for any random codeword $C$ chosen from $\mathcal{C}_{q}^{Q A M}$,

$$
\operatorname{Pr}\left\{\operatorname{PMEPR}_{C_{q}^{Q A M}}(C) \geq \log n+4 \log \log n\right\} \leq O\left(\frac{1}{\log ^{4} n}\right)
$$

Proof: Let's define the real function $s_{C}(\alpha, \theta)$ as,

$$
s_{C}(\alpha, \theta)=\operatorname{Re}\left\{e^{j \alpha} s_{C}(\theta)\right\}
$$

As pointed out in [9], this definition enables us to deal with the maximum of a real function and generalize the result of Halasz. Let's first define

$$
K=\max _{\alpha, \theta}\left|s_{C}(\alpha, \theta)\right|=\max _{\theta}\left|s_{C}(\theta)\right|
$$


We then define a function $0 \leq u(x) \leq 1$ as,

$$
u(x)= \begin{cases}0 & |x| \leq M \\ 1 & |x| \geq M+\Delta\end{cases}
$$

where $E_{a v}=E\left\{\left|c_{i}\right|^{2}\right\}, \Delta=\sqrt{n / \log n}$, and $M=$ $\sqrt{n E_{a v} \log n+O(\log \log n)}$. We also assume $u(x)$ is a function which is 10 times differentiable such that $u^{(r)}(x)=O\left(\Delta^{-r}\right)$ for $1 \leq r \leq 10$. Consequently, we define the random variable $\eta$ as,

$$
\begin{aligned}
\eta & =\int_{0}^{2 \pi} \int_{0}^{2 \pi} u\left(s_{C}(\alpha, \theta)\right) d \theta d \alpha \\
& =\int_{0}^{2 \pi} \int_{0}^{2 \pi} \int_{-\infty}^{\infty} e^{j t u\left(s_{C}(\alpha, \theta)\right)} v(t) d t d \theta d \alpha
\end{aligned}
$$

where $v(t)$ is the Fourier transform of $u(x)$. On the other hand, using Bernstien inequality [4], and the definition of $\eta$, we can deduce that if $\eta<\frac{1}{n \log ^{2} n}$, this implies that $K \leq M+2 \Delta[9],[11]$. Therefore,

$$
\operatorname{Pr}\{K \leq M+2 \Delta\} \geq \operatorname{Pr}\left\{\eta \leq \frac{1}{n \log ^{2} n}\right\}
$$

By computing the mean of the random variable $\eta$, we can then use the Markov inequality to find a lower bound for Eq. (10). This is derived in [10] and by letting $M=$ $\sqrt{n E_{a v} \log n+O(\log \log n)}$, it is shown that

$$
\operatorname{Pr}\left\{\eta \leq \frac{1}{n \log ^{2} n}\right\} \geq 1-O\left(\frac{1}{\log ^{4} n}\right)
$$

theorem follows from (10) and (11).

It is shown in [10] that the asymptotic analysis in Theorem 1 boils down to the computation of the characteristic function of $s_{C}(\theta)$. For the PSK constellation, we can use a similar argument to that of the QAM case since the $c_{i}$ 's are independent. However, for spherical codes, the $c_{i}$ 's are not independent anymore, and we instead use the following lemma that derives the characteristic function of $s_{C}(\theta)$ when $C$ is uniformly distributed over $\Omega_{n}$. The same analysis goes through for computing the characteristic function of $s_{C}(\alpha$, theta $)$.

Lemma 1. Let $C=\left(c_{1}, \ldots, c_{n}\right)$ be a random complex vector uniformly distributed over $\Omega_{n}$ and $s_{C}(\theta)=$ $\sum_{i=1}^{n} c_{i} e^{j \theta i}$. Then

$$
E\left\{e^{j k \varepsilon_{C}(\alpha, \theta)}\right\}=\frac{2^{n} \Gamma(n)}{k^{n} n^{n-1}} J_{n}(n k)
$$

Proof. The proof relies on the fact that the vector $\left(c_{1}, \ldots, c_{n}\right)$ isotropically distributed and so its distribution remains the same under rotation or multiplication by uni- tary matrices. Therefore,

$$
\begin{aligned}
p\left(s_{C}(\theta)\right) & =p\left(<\left(c_{1}, \ldots, c_{n}\right),\left(e^{j \theta}, \ldots, e^{j n \theta}\right)>\right) \\
& =p\left(<U\left(c_{1}, \ldots, c_{n}\right),(1,0, \ldots, 0)>\right) \\
& =p\left(\sqrt{n} c_{1}\right)
\end{aligned}
$$

since we can premultiply the Vandermonde vector by a unitary matrix, $U$, and rotate the vector s.t. $U\left(e^{j \theta}, \ldots, e^{j n \theta}\right)=(\sqrt{n}, 0, \ldots, 0)$ without changing the distribution of $C$. It can be further shown that by letting $c_{i}=r_{i} e^{j \phi_{i}}$, then $r_{1}$ and $\phi_{1}$ are independent with the following distribution,

$$
\begin{aligned}
& p\left(r_{1}\right)=\frac{2}{n^{n-1}} r_{1}\left(n-r_{1}^{2}\right)^{n-1}, \\
& p\left(\phi_{1}\right)=\frac{1}{2 \pi}
\end{aligned}
$$

The lemma follows by using (13) and (14).

We can now use the asymptotic expansion of Bessel functions to write (12) as,

$$
E\left\{e^{j k s_{C}(\alpha, \theta)}\right\}=e^{-n\left(k^{2} / 2+\alpha k^{4}+O\left(k^{6}\right)\right)}(1+O(1 / n))
$$

which allows us to generalize the proof for the QAM case to spherical codes as well.

\subsection{Lower Bound}

Theorem 2 obtains the probability of having PMEPR slightly less than $\log n$ for QAM, PSK, and spherical codes and shows that this probability goes to zero as $n$ tends to infinity.

Theorem 2 (Lower Bound-QAM Case). Let $s_{C}(\theta)=$ $\sum_{i=1}^{n} c_{i} e^{j 2 \pi \theta i}$ where $c_{i}=a_{i}+j b_{i}$ and the $a_{i}$ 's and $b_{i}$ 's each have i.i.d and even distributions. Also, let $\mathcal{C}_{q}^{Q A M}$ be the ensemble of all the admissible codewords. Then, for large values of $n$, and for any random codeword $C$ chosen from $\mathcal{C}_{q}^{Q A M}$,

$$
\operatorname{Pr}\left\{\operatorname{PMEPR}_{C_{q}^{Q A M}}(C) \leq \log n-6.5 \log \log n\right\} \geq O\left(\frac{1}{\log ^{4} n}\right)
$$

Proof: The proof is similar to the upper bound without using the parameter $\alpha$. Since we are looking for a lower bound for $\operatorname{Pr}\left\{\operatorname{PMEPR}_{\mathcal{C}}(C)>\lambda\right\}$, instead of considering the maximum of $s_{C}(\theta)$ over all $\theta$, we may consider the maximum of $s_{C}^{R}(\theta)=\Re\left\{s_{C}(\theta)\right\}$ over its $n$ samples $\theta_{i}=$ $\pi(2 i+1) / n$ for $i=1, \ldots, n$. We also again define $u(x)$ 
as in Theorem 1. Then the random variable $\eta$ is defined as,

$$
\begin{aligned}
\eta & =\sum_{m=1}^{n} u\left(s_{C}^{R}\left(\theta_{m}\right)\right) \\
& =\sum_{m=1}^{n} \int_{-\infty}^{\infty} e^{j t s_{C}^{R}(\theta)} v(t) d t
\end{aligned}
$$

where we replaced $u(x)$ by its Fourier transform $v(t)$. Now we use the following inequalities,

$$
\begin{aligned}
& \operatorname{Pr}\left\{\max _{0 \leq \theta \leq 2 \pi}\left|s_{C}(\theta)\right| \geq M\right\} \\
& \geq \operatorname{Pr}\left\{\max _{0 \leq m \leq n}\left|s_{C}^{R}\left(\theta_{m}\right)\right| \geq M\right\} \\
&=1-\operatorname{Pr}\{\eta=0\} \\
& \geq 1-\operatorname{Pr}\{\eta=0, \eta \geq 2 E\{\eta\}\} \\
&=1-\operatorname{Pr}\{|\eta-E\{\eta\}|\} \geq E\{\eta\}\} \\
& \geq 1-\frac{\sigma_{\eta}^{2}}{E^{2}\{\eta\}}
\end{aligned}
$$

Therefore, the evaluation of the lower bound boils down to the asymptotic analysis of the first and second moments of $\eta$. It is shown in [10] for $M=$ $\sqrt{n E_{a v} \log n-6.5 n \log \log n}, E\{\eta\} \geq O\left(\log ^{6} n\right)$, and moreover,

$$
\sigma_{\eta}^{2} \leq O\left(E\{\eta\} \log ^{2} n+\log ^{5} n\right)
$$

By replacing the mean and second order of $\eta$ in (19), the theorem follows immediately.

This theorem can be also generalized to symmetric PSK constellations as well as spherical codes by using their characteristic function which is discussed in Eq. (12) [10].

\subsection{Review of The Bounds}

To get better insight into the above results, let $\mathcal{C}_{t}$ corresponds to $\mathcal{C}_{q}^{P S K}, \mathcal{C}_{q}^{Q A M}$, or $\mathcal{C}_{B}$ defined as random codes chosen from symmetric QAM/PSK constellations or random spherical codes. Using the inequality,

$$
\operatorname{Pr}(A)+\operatorname{Pr}(B)-1 \leq \operatorname{Pr}(A \cap B) \leq \operatorname{Pr}(A)
$$

together with Theorems 1 and 2 , we can state the following corollary:

Corollary 1. Let $C$ be a random vector whose entries are chosen independently from a q-ary symmetric constellation including $P S K$ and $Q A M$ or let $C$ be randomly chosen from $\Omega_{n}$ and $\mathcal{C}_{t} \in\left\{\mathcal{C}_{q}^{Q A M}, \mathcal{C}_{q}^{P S K}, \mathcal{C}_{s}\right\}$ be the corresponding ensemble of random codewords. Then, with probability one,

$$
\lim _{n \rightarrow \infty} \frac{\operatorname{PMEPR}_{\mathcal{C}_{t}}(C)}{\log n}=1 \text {. }
$$

\section{Varsharmov-Gilbert Bound for Codes with Bounded PMEPR}

In this section, we use the result of Section 3 to derive a Varsharmov-Gilbert type bound on the rate of code with given minimum distance and PMEPR of less than $\log n$.

Here, we use the Hamming distance between two codewords defined as the number of coordinates in which the two codewords are different,

$$
d_{\min }\left(C^{1}, C^{2}\right)=\left|\left\{i: c_{i}^{1} \neq c_{i}^{2}, i=1, \ldots, n\right\}\right|,
$$

and minimum distance of $\mathcal{C}$ is the minimum of (22) over all codewords $C^{1} \neq C^{2}$. The rate of the code family, $\mathcal{C}$, is also defined as,

$$
R=\frac{1}{n} \log _{q}|\mathcal{C}|
$$

where $|\mathcal{C}|$ is the cardinality of the set $\mathcal{C}$.

Theorem 3. Let $\mathcal{Q}_{q}$ be a complex $q$-ary symmetric $P S K$ or $Q A M$ constellation, $R>0$, and $0 \leq \delta \leq \frac{q-1}{q}$, if we have

$$
R \leq 1-H_{q}(\delta)-O\left(\frac{1}{n}\right)
$$

then, asymptotically, there exists a code $\mathcal{C}$ of length $n$, with entries chosen from $\mathcal{Q}_{q}$, rate $R$, minimum Hamming distance $d_{\min }=\lfloor\delta n\rfloor$, and $\mathrm{PMEPR}_{\mathcal{C}}<\log n+4 \log \log n$.

Proof: For the construction of the code, we use the Varsharmov-Gilbert argument as follows. Clearly, we have $q^{n}$ number of codewords in which $n_{\text {bad }}=$ $q^{n} \operatorname{Pr}\left\{\operatorname{PMEPR}_{\mathcal{C}_{q}}(C)>\log \left(n \log ^{4} n\right)\right\}$ number of them have PMEPR of greater than $\log \left(n \log ^{4} n\right)$. Moreover we define $V_{q}(n, t)$ as the maximum number of $q$-ary $n$ dimensional codewords within Hamming distance $t$ of a codeword which is equal to $\sum_{i=1}^{t}\left(\begin{array}{l}n \\ k\end{array}\right)(q-1)^{i}[12]$.

Now we construct the code $\mathcal{C}$ by first eliminating the $n_{\text {bad }}$ codewords with undesired PMEPR from the $q^{n}$ codewords, and then we select a codeword from the remaining codewords and eliminate all codewords within distance $d_{\text {min }}=\delta n$ which is at most $V_{q}(n,\lfloor\delta n\rfloor)$. Therefore, as. long as we have the following inequality

$$
q^{n R} V_{q}(n,\lfloor\delta n\rfloor)+A<q^{n}
$$

we can choose $q^{n R}$ codewords with minimum distance at least $\delta n$ and PMEPR of at most $\log \left(n \log ^{4} n\right)$. We can rewrite $\mathrm{Eq}(25)$ as,

$$
q^{n R} \leq \frac{q^{n}\left(1-\operatorname{Pr}\left\{\operatorname{PMEPR}>\log \left(n \log ^{4} n\right)\right\}\right)}{V_{q}(n,\lfloor\delta n\rfloor)},
$$

Now taking the logarithm of both sides of (26), and using 
$\lim _{n \rightarrow \infty} \frac{1}{n} \log _{q} V_{q}(n,\lfloor\delta n\rfloor)=H_{q}(\delta)[12]$, we get,

$R \leq 1-H_{q}(\delta)+\frac{1}{n} \log _{q} \operatorname{Pr}\left\{\operatorname{PMEPR}<\log \left(n \log ^{\alpha} n\right)\right\}$.

Using Theorem 1 to bound the third term in (27), and using $-\log (1-x)=O(x)$ we can deduce the Theorem.

According to Theorem 3, it follows that not only do there exist spherical high rate codes with PMEPR of $8 \log n$, but there also exist codes chosen from usual constellations like QAM and PSK with the same asymptotic. Similarly, according to the VG bound, we can see that there is no penalty in terms of rate and minimum distance of codes with bounded PMEPR up to $\log n$ if the codewords are chosen from symmetric PSK or QAM constellations.

\section{Future Work and Conclusion}

As mentioned before, multicarrier signals may exhibit PMEPR of order $n$. However, the result of this paper shows that encountering this worst case is highly unlikely. More precisely, asymptotically and in the probability sense, the PMEPR is $\log n$ for a large class of constellations including QAM/PSK symmetric and spherical codes. Now it is not surprising at all to have either spherical codes or codes chosen from QAM/PSK constellations with high rate and large minimum distance and PMEPR of less than $\log n$ [5].

On the other hand, this result does not contradict the fact that there might be exponentially many codewords with constant PMEPR. However the ratio of the number of these codewords to $q^{n}$ has to tend to zero asymptotically. So there still remains an open problem of what is the rate of codes with constant PMEPR? Another issue would be to consider the trade off between rate and minimum distance of codes with bounded PMEPR. We know that the Varsharmov-Gilbert bound remains the same by restricting codewords to PMEPR less than $\log n$. However, what will happen to the rate and minimum distance of a code by reducing the PMEPR to less than $\log n$, e.g. constant?

\section{References}

[1] X. Li and L. J. Cimini, "Effects of clipping and filtering on the performance of OFDM," in Proc. IEEE Veh. Tech. Conf., May 1997, pp. 1634-1638.

[2] S. H. Muller and J. B. Huber, "A comparison of peak power reduction schemes for OFDM" in Proc. IEEE Glob. Comm. Conf., 1997, pp. 1-5.

[3] J. Tellado and J. M. Cioffi, "Efficient aigorithms for reducing PAR in multicarrier systems," in Proc. IEEE Inter. Symp. Info., August 1998, p. 191.

[4] M. Sharif, M. Gharavi-Alkhansari, and B. H. Khalaj, "On the peak to average power of OFDM signals based on oversampling," to appear in IEEE Trans. Comm., 2002.

[5] K .G. Paterson and V. Tarokh, "On the existence and construction of good codes with low peak to average power ratios," IEEE Trans. Inform., vol. 46, no. 6, pp. 1974-1986, Sep. 2000.

[6] J.A. Davis and J. Jedwab, "peak to mean power control in OFDM, Golay complementary sequences, and Reed-Muller codes," IEEE Trans. Inform., vol. 45, no. 7, pp. 2397-2417, Nov. 1999.

[7] H. Ochiai and H. Imai, "On the distribution of the peak to average power ratio in OFDM signals," IEEE Trans. Comm., vol. 49, no. 2, pp. 282-289, Feb. 2001.

[8] M. Sharif and B.H. Khalaj, "Peak to mean envelope power ratio of multicarrier signals: an analytical approach," in Proc. of IEEE ICC, 2001, pp. 1476-1480.

[9] G. Halasz, "On the result of Salem and Zygmund concerning random polynomials," Studia Scien. Math. Hung., pp. 369-377, 1973.

[10] M. Sharif and B. Hassibi, "Asymptotic probability bounds on the peak distribution of complex multicarrier signals and their implications," to be submitted to IEEE Trans. Inform., 2002.

[11] A. Gersho, B. Gopinath, and A. M. Odlyzko, "Coefficient inaccuracy in transversal filtering," The Bell Systems Technical Joumal, vol. 58, no. 10, pp. 23012316, Dec. 1979.

[12] S. B. Wicker, Error control systems for digital communication and storage, Prentice Hall: NJ, Englewood cliffs, 1995. 